\title{
Chaotic Dynamics of Stellar Spin in Binaries and the Production of Misaligned Hot Jupiters
}

\author{
Kassandra R. Anderson ${ }^{1, a}$, Natalia I. Storch ${ }^{1}$, and Dong Lai ${ }^{1}$ \\ Center for Space Research, Department of Astronomy, Cornell University, Ithaca, NY 14853, USA
}

\begin{abstract}
Motivated by observed hot Jupiter systems with high stellar obliquities, we examine the dynamics of stellar spin in planetary systems with distant stellar companions, with inclinations sufficiently high so that the planet undergoes Kozai-Lidov oscillations. In this scenario, the stellar spin axis undergoes complex (and often chaotic) motion. We describe the stellar spin dynamics, highlight the importance of the stellar spin properties, and show that this complex evolution can be preserved in the final distributions of obliquity angles.
\end{abstract}

\section{Introduction}

Many observed hot Jupiter systems show significant misalignments between the spin axis of the host star and the planetary orbital axis. Understanding the origins of these obliquities is helpful in understanding the formation histories of hot Jupiters, and could help differentiate between various proposed migration mechanisms. Several channels exist for producing a misaligned hot Jupiter, including a primordial disk misalignment [2,3,7], planet-planet interactions and scattering [5,13], and Kozai-Lidov oscillations induced by a distant companion $[8,6,12,4,10]$. We focus on the latter scenario, and show that the dynamics of the stellar spin, combined with Kozai-Lidov oscillations, lead to complex (and often chaotic) evolution of the spin-orbit angle $[10,11]$. This complex evolution is manifested in the final distribution of obliquity angles.

\section{Spin-Orbit Evolution}

\subsection{Kozai-Lidov Cycles}

We consider a system consisting of a planet of mass $M_{p}$ around its host star $M_{\star}$ with a distant stellar companion $M_{b}$, where $M_{\star}$ and $M_{b}$ are separated by $a_{b} \sim 100 \mathrm{AU}$. The quadrupole potential of the binary companion causes the planet's orbital angular momentum axis $\hat{\mathbf{L}}$ to precess around the (fixed) binary angular momentum axis $\hat{\mathbf{L}}_{b}$. If $\hat{\mathbf{L}}$ is inclined relative to $\hat{\mathbf{L}}_{b}$ by more than $\sim 40$ degrees, KozaiLidov oscillations in the planet's orbital eccentricity $e$ and inclination $\theta_{\mathrm{lb}}$ are induced, occurring over a timescale

$$
t_{k}=\frac{M_{\star}}{M_{b}}\left(\frac{a_{b}}{a}\right)^{3} \frac{1}{\Omega_{p}},
$$

where $a$ and $a_{b}$ are the semimajor axes of the planet and binary respectively, and $\Omega_{p}$ is the planet's mean motion. $\hat{\mathbf{L}}$ precesses around $\hat{\mathbf{L}}_{b}$ with frequency $\Omega_{\mathrm{pl}}$, where

$$
\Omega_{\mathrm{pl}} \approx \frac{1}{t_{k}\left(1-e^{2}\right)}
$$

\footnotetext{
a e-mail: kanderson@astro.cornell.edu
}

This is an Open Access article distributed under the terms of the Creative Commons Attribution License 4.0, which permits unrestricted use, distribution, and reproduction in any medium, provided the original work is properly cited. 
(see [10] for an exact expression for $\Omega_{\mathrm{pl}}$ ).

\subsection{Stellar Spin Dynamics}

The oblate host star produces a quadrupole moment, causing the stellar spin axis $\hat{\mathbf{S}}$ to precess around the planet's orbital axis $\hat{\mathbf{L}}$ according to

$$
\frac{d \hat{\mathbf{S}}}{d t}=\Omega_{\mathrm{ps}} \hat{\mathbf{L}} \times \hat{\mathbf{S}}
$$

where the spin precession rate is given by

$$
\begin{aligned}
\Omega_{\mathrm{ps}} & =-\frac{3 G M_{p}\left(I_{3}-I_{1}\right)}{2 a^{3}\left(1-e^{2}\right)^{3 / 2}} \frac{\cos \theta_{\mathrm{sl}}}{S} \\
& \propto \frac{M_{p} \Omega_{\star}}{a^{3}\left(1-e^{2}\right)^{3 / 2}} .
\end{aligned}
$$

This spin precession, coupled with Kozai-Lidov (KL) oscillations leads to a wide range in dynamical behavior, depending on the relative values of the precession rates $\Omega_{\mathrm{pl}}$ and $\Omega_{\mathrm{ps}}$. Note that both $\Omega_{\mathrm{ps}}$ and $\Omega_{\mathrm{pl}}$ are strong functions of eccentricity. Since the eccentricity can change from $e \sim 0$ to $e \sim 1$ throughout a KL cycle, the ratio $\Omega_{\mathrm{ps}} / \Omega_{\mathrm{pl}}$ can vary widely.

We thus expect three qualitatively different regimes: If $\Omega_{\mathrm{ps}} / \Omega_{\mathrm{pl}}<1$ throughout the KL cycle, the spin axis $\hat{\mathbf{S}}$ precesses around $\hat{\mathbf{L}}$ much more slowly than $\hat{\mathbf{L}}$ precesses around the binary axis $\hat{\mathbf{L}}_{b}$. In such a scenario, the spin axis effectively precesses around the binary axis, so that the spin-binary angle $\cos \theta_{\mathrm{sb}}=\hat{\mathbf{S}} \cdot \hat{\mathbf{L}}_{b} \approx$ constant. We refer to this as the "non-adiabatic regime."

In the opposite regime, the ratio $\Omega_{\mathrm{ps}} / \Omega_{\mathrm{pl}}>1$ throughout the KL cycle. In this scenario, $\hat{\mathbf{S}}$ follows $\hat{\mathbf{L}}$ adiabatically, with spin-orbit angle $\cos \theta_{\mathrm{sl}}=\hat{\mathbf{S}} \cdot \hat{\mathbf{L}} \approx$ constant. For systems that start out with $\hat{\mathbf{S}}$ and $\hat{\mathbf{L}}$ aligned, the spin-orbit angle remains small. We refer to this as the "adiabatic regime."

Finally, if $\Omega_{\mathrm{ps}} / \Omega_{\mathrm{pl}} \approx 1$ at some point during the KL cycle, a secular resonance occurs, and the stellar spin axis undergoes chaotic motion, leading to complex behavior (see [11] for a discussion of the origin of the chaos). We refer to this as the "trans-adiabatic regime." An example showing the chaotic spin evolution of two systems with nearly identical initial conditions is presented in Figure 1, taken from [10].

The ratio $\Omega_{\mathrm{ps}} / \Omega_{\mathrm{pl}}$ has the dependence

$$
\frac{\Omega_{\mathrm{ps}}}{\Omega_{\mathrm{pl}}} \propto \frac{M_{p} \Omega_{\star}}{a^{9 / 2}}
$$

where $M_{p}$ is the planet mass, $\Omega_{\star}$ is the stellar rotation rate and $a$ is the planet's semimajor axis. Thus, systems with rapidly rotating stars, massive planets, and small semimajor axes tend to be more adiabatic. Note that due to the strong dependence on semimajor axis, all migrating planets that result in hot Jupiters end up in the adiabatic regime, so that the final spin-orbit angle freezes to a constant value, as shown in Figure 2.

\section{Observational Consequences}

Given the three qualitatively different regimes and the potential for chaotic evolution of the stellar spin axis, we now adopt a population synthesis approach, and examine the final distributions of spinorbit misalignment angles for hot Jupiters undergoing KL oscillations (to appear in [1]). We include the effects of tidal dissipation in the planet and extra pericenter precession due to short range forces (see [10] for further details). We focus on three planet masses $\left(M_{p}=1,3,5 M_{J}\right)$, a range of initial planetary semimajor axes $(a=1.5-3.5 \mathrm{AU})$, and two different host star masses $\left(M_{\star}=1.0\right.$ and 


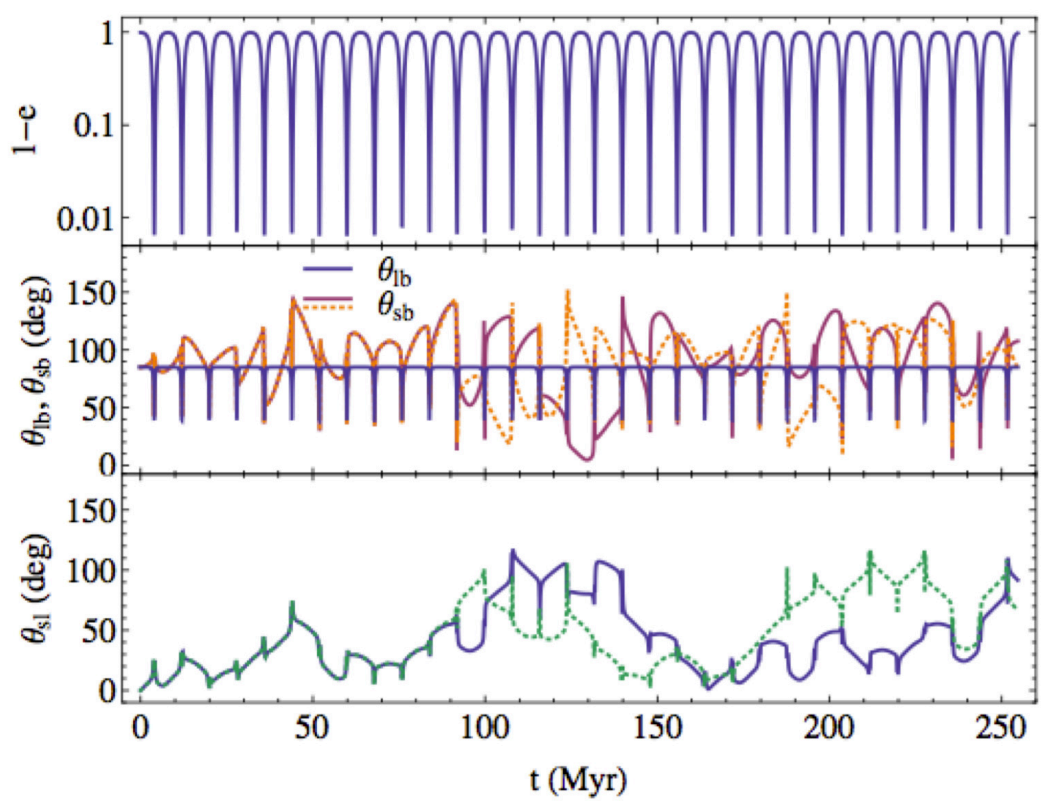

Fig. 1: From [10]: Evolution of the eccentricity (top panel), inclination $\theta_{\mathrm{lb}}$ and spin-binary angle $\theta_{\mathrm{sb}}$ (middle), and spin-orbit angle $\theta_{\mathrm{sl}}$ (bottom) for two systems with nearly identical initial conditions. Although the evolution for the orbital elements is nearly identical between the two systems, the chaos in $\theta_{\mathrm{sl}}$ is clearly demonstrated.

$1.4 M_{\odot}$, corresponding to $\mathrm{G}$ and $\mathrm{F}$ type stars), each with different spin evolution. In addition to spin precession, we allow the star to slow down via magnetic braking, using the Skumanich law, where the spin-down rate takes the form $d \Omega_{\star} / d t=-\alpha \Omega_{\star}^{3}$, and the parameter $\alpha=1.5 \times 10^{-14} \mathrm{yr}$ for a solar-type (G) star, but is a factor of 10 smaller for a more massive (F) star.

Figure 3a shows the resulting distributions of $\theta_{\mathrm{sl}}$ for the $\mathrm{G}$ star. For planet masses $M_{p}=1 M_{J}$, the distribution is bimodal, in agrement with previous work. As the planet mass increases to $M_{p}=3 M_{J}$, the number of systems with small obliquities increases, because the stellar spin is more strongly coupled to the orbit for larger planet masses (adiabatic evolution). Finally, for $M_{p}=5 M_{J}$, most systems result in alignment, because the spin precession is even more adiabatic.

In contrast, the distributions are quite different for the F star, shown in Figure $3 b$. The number of highly misaligned systems decreases with increasing planet mass, but the distribution remains bimodal even for large $\left(5 M_{J}\right)$ planets. Comparing Figure $3 \mathrm{a}$ and $3 \mathrm{~b}$ shows that the final distribution of spin-orbit misalignment angles depends strongly on the stellar spin properties and planet mass, to be presented in greater detail in [1].

\section{References}

1. Anderson, K. R., Storch, N. I., \& Lai, D. 2014, in preparation

2. Bate, M. R., Lodato, G., \& Pringle, J. E. 2010, MNRAS, 401, 1505

3. Batygin, K., \& Adams, F. C. 2013, ApJ, 778, 169

4. Fabrycky, D., \& Tremaine, S. 2007, ApJ, 669, 1298

5. Ford, E. B., \& Rasio, F. A. 2008, ApJ, 686, 621

6. Kozai, Y. 1962, AJ, 67, 591

7. Lai, D. 2014, MNRAS, 440, 3532 

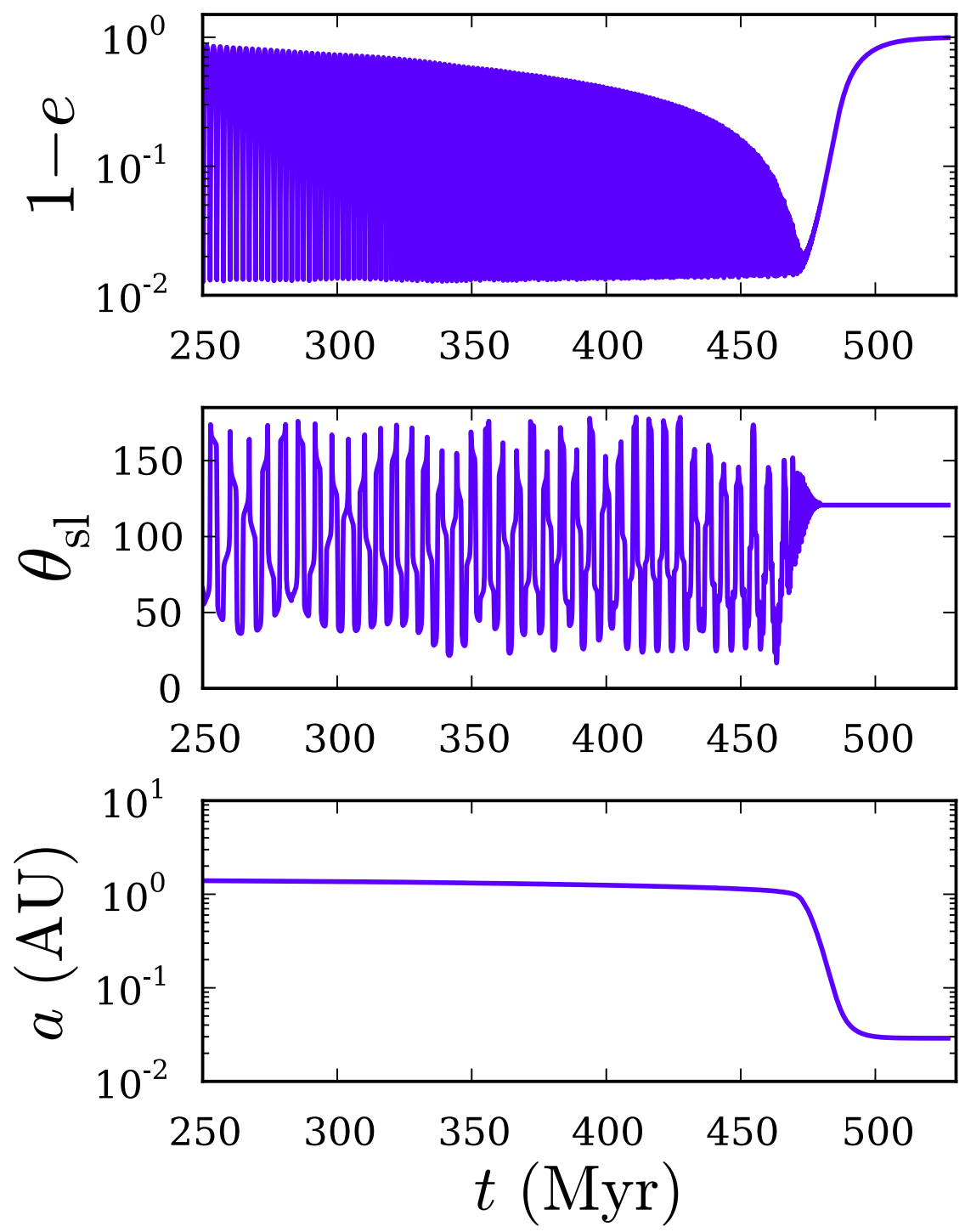

Fig. 2: Time-evolution of a system with tidal dissipation, highlighting the rapid "freezing" of the spinorbit angle, as the system transitions from the trans-adiabatic regime to the fully adiabatic regime.

8. Lidov, M. L. 1962, Planet. Space. Sci., 9, 719

9. Mazeh, T., \& Shaham, J. 1979, A \& A, 77, 145

10. Storch, N. I., Anderson, K. R., \& Lai, D. 2014, Science, 345, 1317

11. Storch, N. I., \& Lai, D. 2014, submitted

12. Wu, Y., \& Murray, N. 2003, ApJ, 589, 605

13. Wu, Y., \& Lithwick, Y. 2011, ApJ, 735, 109 


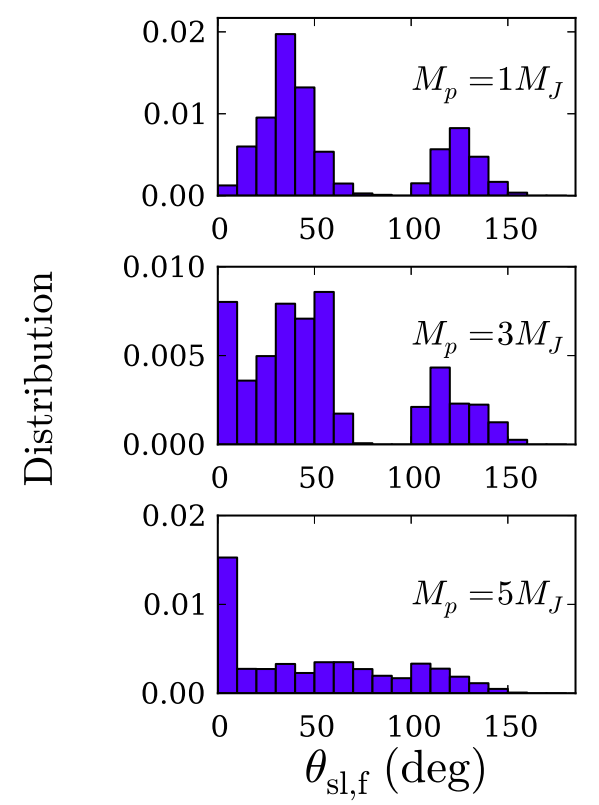

(a) $M_{\star}=1.0 M_{\odot}$

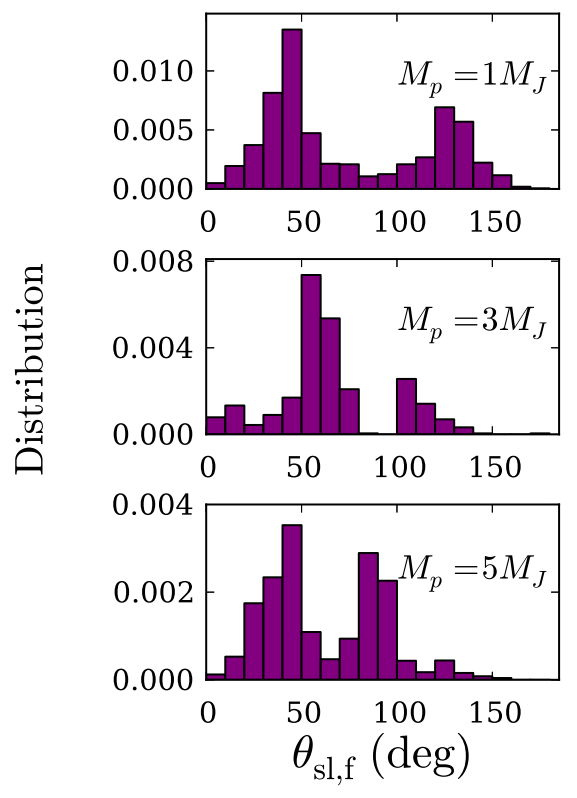

(b) $M_{\star}=1.4 M_{\odot}$

Fig. 3: Distributions of final obliquity angles for a host star of $M_{\star}=1.0 M_{\odot}$ (left) and $M_{\star}=1.4 M_{\odot}$ (right). The solar-mass star has magnetic braking parameter $\alpha=1.5 \times 10^{-14} \mathrm{yr}$ (calibrated to the Sun), and the massive star has $\alpha=1.5 \times 10^{-15} \mathrm{yr}$ to account for the fact that massive stars tend to spin more rapidly. The distributions of $\theta_{\mathrm{sl}, \mathrm{f}}$ depend strongly on the planet mass and stellar spin properties. 